UCB-PTH-05/41

LBNL-59212

\title{
Taming the Runaway Problem of Inflationary Landscapes
}

\author{
${ }^{a}$ Lawrence J. Hall, ${ }^{a}$ Taizan Watari, and ${ }^{b}$ T. T. Yanagida \\ ${ }^{a}$ Department of Physics and Lawrence Berkeley National Laboratory, \\ University of California, Berkeley, CA 94720, USA \\ ${ }^{b}$ Department of Physics, University of Tokyo, Japan
}

\begin{abstract}
A wide variety of vacua, and their cosmological realization, may provide an explanation for the apparently anthropic choices of some parameters of particle physics and cosmology. If the probability on various parameters is weighted by volume, a flat potential for slowroll inflation is also naturally understood, since the flatter the potential the larger the volume of the sub-universe. However, such inflationary landscapes have a serious problem, predicting an environment that makes it exponentially hard for observers to exist and giving an exponentially small probability for a moderate universe like ours. A general solution to this problem is proposed, and is illustrated in the context of inflaton decay and leptogenesis, leading to an upper bound on the reheating temperature in our sub-universe. In a particular scenario of chaotic inflation and non-thermal leptogenesis, predictions can be made for the size of $\mathrm{CP}$ violating phases, the rate of neutrinoless double beta decay and, in the case of theories with gauge-mediated weak scale supersymmetry, for the fundamental scale of supersymmetry breaking.
\end{abstract}




\section{Introduction}

Our complicated world is based on hundreds of elements that have different properties. The hundreds of elements reflect the existence of hundreds of stable nuclei. The variety of stable nuclei requires fine choices of parameters such as $\alpha_{\mathrm{QCD}}, \alpha_{\mathrm{QED}}, m_{u}, m_{d}$ and $m_{e}$. Even a deviation of a few $\%$ in $\alpha_{\mathrm{QCD}}$ from the current value could destabilize or stabilize such nuclei as ${ }^{2} \mathrm{H},{ }^{2} \mathrm{He}$ and di-neutrons, completely changing the thermonuclear processes, chemical abundances of the universe and lifetime of stars [1. Such fine tuning cannot be explained by the naturalness principle. Rather, it is easily explained by a combination of the many-universe idea (a cosmological diversity in the choice of theories and their parameters) with anthropic selection [2, 3]. It is only in the sub-universes with fine-tuned parameters that observers made of many species of atoms exist. This combination also provides a simple solution to both the why-small and why-now problems of the cosmological constant [4, 5].

Once we accept that the combination of many-universes with anthropic selection plays a role in determining the values of observed parameters, then we need to change our way of thinking. The combination not only confirms that the observed values of parameters are consistent with the existence of observers, but, because the notion of naturalness is replaced by probability, it changes to some extent the questions that should be asked. The fine tuning of parameters and initial conditions of inflation is one prominent example: if the probability distribution is weighted by the volume of the sub-universes, such a fine tuning is rather probable, as long as theory space allows the inflaton mass to scan [6], or provided appropriate initial conditions

are realized somewhere in the universe [7. We call such a space of theories containing lots of inflationary regions an inflationary landscape.

The problem of inflationary landscapes [8, 9] is that we are no longer able to choose the parameters of the inflation model by hand to fit the observed data; rather these parameters (and consequently the normalization of the density perturbations) are predicted by a combination of statistics from the landscape, cosmological dynamics and anthropic conditions [3. In particular, it is generically true that as the inflaton mass becomes smaller, so the inflated volume becomes exponentially larger. This exponential behaviour of the volume-weighted probability distribution implies not only that a small inflaton mass is very probable [6], but also that the inflaton mass will become as small as possible until it is prevented by some anthropic reason. Thus, a naive prediction of inflationary landscapes would be that the inflaton mass is so small that the environment is exponentially hard for observers to exist [8] because, for example, the density perturbations are too small or too large (this was called " $\sigma$ problem" in [8] ), the baryon 
asymmetry is too small or there is not enough matter. Whatever the reason, this picture apparently does not lead to our universe. ${ }^{1}$ Since the slow-roll volume factor selects vacua with smaller and smaller inflaton mass, we call this the inflation runaway problem.

The $\sigma$ problem may be solved [8, 9] if the density perturbations arise from fluctuations of light fields other than the inflaton [11, 12. Even then, the inflaton mass has to be predicted at a moderate value, or otherwise, there could $b^{2}$ the same problem as above, for instance, in the baryon asymmetry or dark matter abundance. Thus, this is quite a generic problem of inflationary landscapes. Note, however, that the probability distribution on observable parameters depends sensitively on the boundary values of unobservable parameters and amplitudes being scanned. Thus, the distribution is not exponentially sensitive to observables [13] in some landscapes where the observable-unobservable correlation is sufficiently week 8 .

In this article, we present an idea of how to stop the runaway behaviour of the inflaton mass, along with its concrete implementation. In section 2.1 we introduce the runaway problem, and in section 2.2 we point out a caveat that allows a solution. It is crucial to distinguish anthropically relevant parameters from fundamental parameters, and further

(a) to have a sharp function, or threshold behaviour, of the former in terms of the latter, and

(b) to have an anthropic reason that exponentially disfavours some range of relevant parameters.

The runaway problem can be overcome when both (a) and (b) are satisfied. In section 2.3, we see that (a) can be realized as the reheating temperature $T_{R}$ depends upon the inflaton mass. If the inflaton decays dominantly to two heavy particles, $T_{R}$ drops rapidly as the inflaton mass becomes close to twice of the mass of the heavy particle. The inflaton mass is predicted to be close to twice the mass of the heavy particle. In this article we assume that only the inflaton mass and the cosmological constant are scanned in the landscape, not the mass of the heavy particle. ${ }^{3}$ Section 3 explains how $T_{R}$ can be a relevant parameter. If the baryon asymmetry is generated by thermal leptogenesis, a very low reheating temperature leads to a low baryon asymmetry and, if the baryon asymmetry is too low, galaxies cannot form before protons decay. Thus, too low a $T_{R}$ is exponentially disfavoured. Therefore, both (a) and (b) can be realized, and the runaway problem is solved. In section 4 we study the possibility that the heavy particles

\footnotetext{
${ }^{1}$ After understanding astrophysics and biology better, however, the naive prediction may turn out to be the solution to Fermi's paradox "Where is everybody?" [10.

${ }^{2}$ When the inflaton mass does not affect late time cosmology except for the number of e-foldings, as in the hybrid inflation model in [8], such a problem does not exist.

${ }^{3} \mathrm{We}$ do so as a first attempt to address the runaway problem in the simplest and easiest situation. There may be room to lift some of these strong assumptions, but this is beyond the scope of this article.
} 
produced by inflaton decay are identified with one of the right-handed neutrinos, hoping for constraints and predictions in the neutrino sector. The last section is devoted to conclusions and discussion, together with a summary of our predictions. The appendix investigates one of the uncertainties in the analysis in sections 3 and 4 , the effect of evaporation of particles from proto-galaxies.

\section{The Runaway Problem and How to Solve It}

\subsection{The Inflation Runaway Problem}

The probability distribution $d \mathcal{P}(\xi) / d \xi$ describes the fraction of observers in the overall universe who are in sub-universes where the fundamental parameters, $\xi$, are in the range $\xi$ to $\xi+d \xi[6]$.

$$
d \mathcal{P}(\xi)=d \xi I(\xi) \mathcal{V}(\xi) \mathcal{A}(\alpha(\xi))
$$

The initial volume distribution $I(\xi)$ includes the density of vacuum states and the probability distribution for the initial amplitudes of the inflaton field. The density of states can be, in principle, calculated from a given landscape, or from a top-down theory [14]. The volume factor $\mathcal{V}(\xi)$ [15, 16] accounts for the expansion of each sub-universe during and after slow-roll inflation

$$
\mathcal{V}(\xi) \propto e^{3 N_{e}(\xi)}
$$

With these definitions, any volume expansion of the universe prior to the slow-roll stage is incorporated in the initial factor $I(\xi)$. For instance, if a landscape supports eternal inflation, its volume expansion is to be incorporated in the initial factor $I(\xi)$. The anthropic factor $\mathcal{A}(\alpha)$ depends only on parameters $\alpha$ of the low energy effective theory and of late time cosmology. We call these the relevant parameters, some of which are relevant for providing an environment suitable for observers. Ultimately they depend on the fundamental parameters, $\alpha(\xi)$. The anthropic factor vanishes for many values of the relevant parameters, for example $\mathcal{A}\left(m_{e}=\right.$ $5 \mathrm{MeV})=0$. However, in this paper the only aspect of the anthropic factor that we consider is the formation of galaxies having an appropriate number of baryons, since we pay attention only to the scanning of inflation parameters.

It is rather likely that a landscape supports some eternally inflating regions [17, 15, 18]; our standard-model vacuum with a minute cosmological constant may result from one of them. An eternally inflating vacuum can tunnel or jump into regions of the landscape that support slow-roll inflation through bubble nucleation or through quantum fluctuation of inflaton fields, 
eventually ending up in a radiation dominated standard-model universe after reheating (c.f. [19]). The volumes of eternally inflating vacua keep expanding forever, and hence the volume created through nucleation or fluctuation is infinite as well. There is an ongoing effort to regularize these infinities so that the probability distribution (10) is well-defined [16, 20, 9, 21]. In this article, we assume that such a regulation is possible. We further assume ${ }^{4}$ that, when the probability distribution is made well-defined, the classical volume expansion factor (2) is not exactly cancelled by $I(\xi)$. Such a cancellation appears unlikely, and one of our motivations to consider the scanning of some inflation parameters is to seek an origin for a flat inflaton potential in the volume-weighted probability distribution.

Since the number of e-foldings of slow-roll inflation $N_{e} \gtrsim 60$ for our sub-universe, $\mathcal{V}(\xi)$ ensures that the probability distribution involves a powerful exponential dependence on the parameters of the inflation potential [3]. After integrating over all fundamental parameters and initial amplitudes of various fields that we cannot observe directly, $\xi_{\|}$, one obtains the probability distribution of relevant parameters (fraction of observers who see them)

$$
d \mathcal{P}(\alpha)=d \alpha\left[\int d \xi_{\|}\left(d \alpha / d \xi_{\perp}\right)^{-1} I(\xi) \mathcal{V}(\xi)\right] \mathcal{A}(\alpha) .
$$

Within the anthropic window, where the anthropic factor is changing only mildly, the overall probability distribution is governed by the factor in the square bracket. Unless there is an accidental cancellation between $I(\xi)$ and $\mathcal{V}(\xi)$, or unless the region of integration determined by a landscape is finely designed, ${ }^{5}$ the distribution of observables is generically exponentially sensitive to inflation-related quantities such as the density perturbations and the reheating temperature [8]. An immediate consequence is that an exponentially small fraction of observers in the universe would see values of these parameters in the middle of the anthropic window 8, 9]. Since we do not accept this conclusion that our sub-universe is so extraordinarily special, there must be something wrong with these assumptions or arguments.

In fact, the exponential behaviour from the volume expansion factor $\mathcal{V}(\xi)$ is so steep that the first question to be answered is whether the overall probability distribution can be normalized. If $\mathcal{I}(\xi)$ and $\mathcal{A}(\xi)$ are power-law functions, the probability distribution grows forever and is not well-defined. At a minimum, the exponential behaviour must be cut off so that observable

\footnotetext{
${ }^{4}$ Another assumption that we make in this article is that the volume distribution $I(\xi) \mathcal{V}(\xi) d \xi$ can be treated as a continuous distribution over field theory parameters $\xi$. This assumption may be translated into a sufficiently dense landscape of vacua, or bubble tunneling rates without large hierarchy among them; for more information, see $[8]$.

${ }^{5} \mathrm{~A}$ landscape approximated by an ensemble of chaotic inflation potentials, with a mass-independent cut off on the maximum field value, is such a possibility [8]. Reference [13] presents an explicit realization.
} 
parameters have average values that are well-defined. This may be accomplished in three different ways.

If only a limited number of states are available in a landscape after imposing rigid anthropic conditions, ${ }^{6}$ the probability distribution is always normalizable. On the other hand, the most probable vacuum among them does not necessarily satisfy non-rigid anthropic conditions [8].

Another possibility is that the initial volume factor provides another exponential distribution, so that the combined volume distribution $I(\xi) \mathcal{V}(\xi) d \xi$ has two exponentials counteracting each other. Various properties of the most probable vacuum will be determined by how the two exponential distributions balance. Without studying landscapes of the fundamental theory (including eternally inflation regions), it may be hard to understand why the probable vacuum, supposed to describe our universe, happens to fall inside the anthropic window.

The other possibility is that the probability is rendered well-defined by an anthropic factor that provides an exponential suppression. Instead of relying on the initial distribution, about which we know very little, a special form for the anthropic factor is required, offering the hope of observable consequences. We find this case rather attractive and pursue it in this article. The problem of this possibility, however, is that it is only outside the anthropic window where the anthropic factor begins to decrease and counter the exponential growth in the inflation volume. If we assume that both the volume factor $I(\xi) \mathcal{V}(\xi) \approx e^{3 N_{e}\left(\xi_{\perp}\right)}$ and the anthropic factor $\mathcal{A}(\alpha(\xi)) \approx e^{-F(\alpha(\xi))}$ are governed by some power functions $N_{e}(\xi)$ and $F(\alpha(\xi))$, then the peak of the probability distribution lies roughly where

$$
\frac{\partial N_{e}\left(\xi_{\perp}\right)}{\partial \ln \xi_{\perp}}-\frac{\partial F\left(\alpha\left(\xi_{\perp}\right)\right)}{\partial \ln \xi_{\perp}} \approx 0, \quad \text { and hence } \quad N_{e}\left(\xi_{\perp}\right) \approx F\left(\alpha\left(\xi_{\perp}\right)\right) .
$$

This implies that the majority of observers in the universe live in sub-universes having an environment exponentially hard for observers to exist: $\mathcal{A} \approx e^{-F(\alpha(\xi))} \lesssim e^{-\mathcal{O}(100)} \ll 1$. The powerful exponential factor $\mathcal{V}(\xi)$ pushes the expectation value of observables outside the anthropic window (see Fig. 11) [8]. ${ }^{7}$ We call this the inflation runaway problem.

\footnotetext{
${ }^{6}$ It should be noted that an anthropic factor from cosmological origins tends not to provide a rigid cut-off in the range of parameters [22, 8]. Any conditions that involve density perturbations fall into this category. This is because Lagrangian parameters (or sub-universes) determine only the standard deviation $\sigma$ of density fluctuations $Q$, whereas the conditions are imposed on the real fluctuations $Q$. The anthropic factor $\mathcal{A}(\sigma)$ can be calculated from $\widetilde{\mathcal{A}}(Q)$ by assuming a Gaussian distribution:

$$
\mathcal{A}(\sigma) \approx \int d Q e^{-(Q / \sigma)^{2}} \widetilde{\mathcal{A}}(Q)
$$

Thus, at most the anthropic factor can provide an exponential suppression.

${ }^{7}$ This problem persists even if density perturbations are generated by the curvaton [1] or modulated reheat-
} 


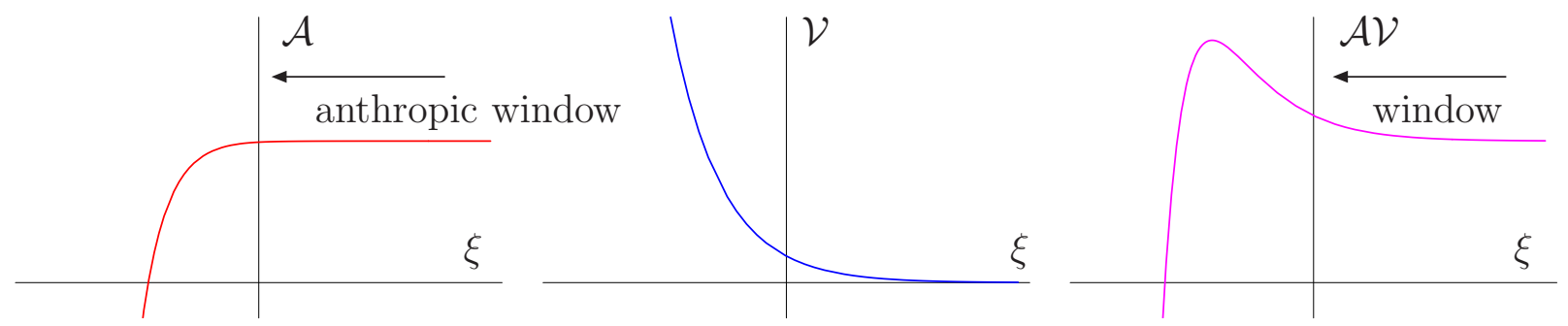

Figure 1: Schematic picture of the inflation runaway problem. From left to right: the anthropic factor $\mathcal{A}$, the volume factor $\mathcal{V}$, and the combined probability distribution $\mathcal{V} \mathcal{A}$, all on a logarithmic scale. Normalization of each factor is arbitrary, and hence there is no importance in the absolute height in the figure. The region to the right of the vertical axis is the anthropic window, and the peak of the probability distribution lies outside the window.

\subsection{An Idea to Solve the Problem}

There is a caveat in the above argument that we exploit in this article. The volume expansion factor is a function of the fundamental parameters of inflation models $\xi$, whereas the anthropic factor depends on the relevant parameters, $\alpha(\xi)$. The caveat is in the map between them: what if the map shows a very sharp behaviour?

Let us assume that $\alpha(\xi)$ has a region where it is rapidly varying, i.e., $\left|\alpha^{\prime}\right| \equiv\left|d \alpha / d \xi_{\perp}\right|$ is large, as shown in Fig. 2. Then the factor $\left(d \alpha / d \xi_{\perp}\right)^{-1}$ in (3) becomes small as parameters enter this region, as illustrated by a green curve in Fig. 3 and the volume factor $\mathcal{V}(\xi)$ becomes a mild function of $\alpha$ (blue curve in Fig. 3). Although $\mathcal{V}$ is exponentially sensitive to $\xi$, the dependence on $\alpha$ in this region is much weaker. Thus, the factor in the square brackets in (3) can form a mild peak, as shown in Fig. 3. If this particular region of $\alpha$ has an overlap with the anthropic window, where $\mathcal{A}(\alpha)$ depends only mildly on $\alpha$, the combined probability distribution may have a mild peak inside the anthropic window, as shown in the right of Fig. 3. This explains how inflationary landscapes can predict average values of observables inside the anthropic window.

In this case, the difficulty in (4) is avoided in the following way. The derivative of the distribution function in (3) has to be zero somewhere in the anthropic window, forming a peak

ing 12 mechanism, so that their normalization is predicted to lie within the window, i.e., the $\sigma$ problem is cured as in [8, 9]. The density perturbations are not the only observables that have to be fixed within moderate values. For example, a sub-universe with a reheating temperature sufficiently low to give an exponentially suppressed anthropic factor is far from the one we observe. 

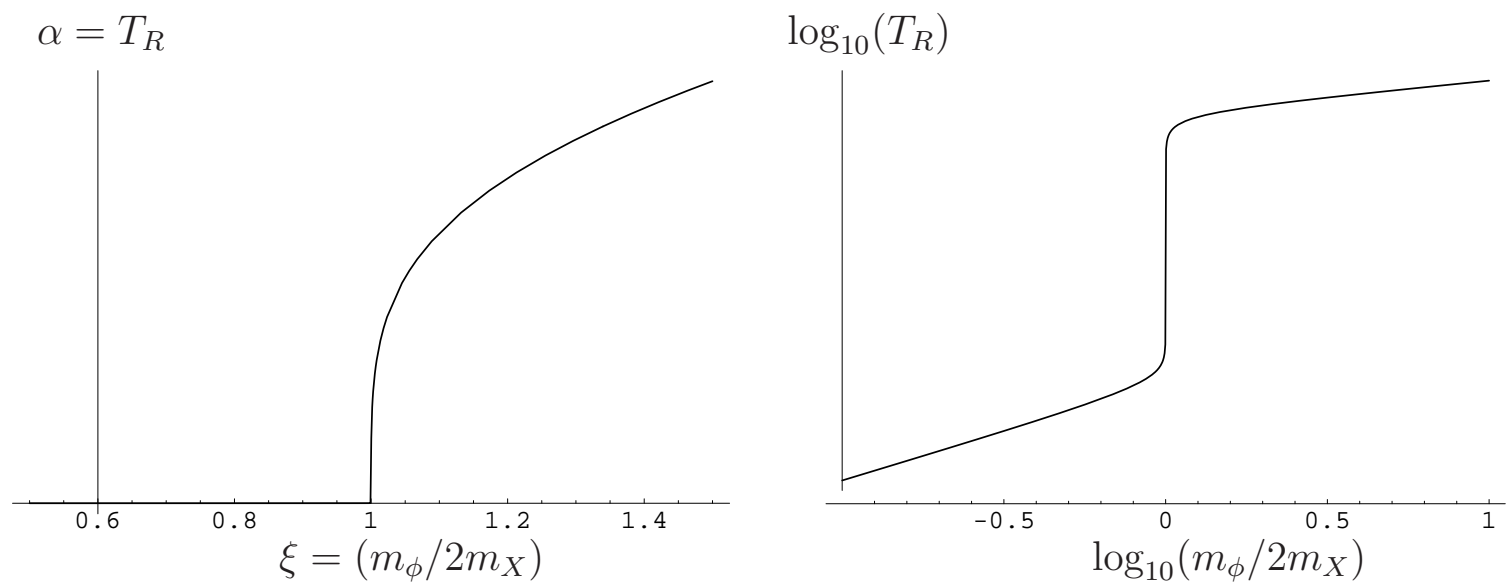

Figure 2: A relevant parameter $\alpha(\xi)$ with a sharp dependence on a fundamental parameter $\xi$. This is interpreted as the dependence of the reheating temperature on the inflaton mass, $T_{R}\left(m_{\phi}\right)$ in section 2.3 , and this figure corresponds to $\lambda^{\prime} \approx 10^{-3}$.

in the distribution. There,

$$
-\frac{\partial \ln \alpha^{\prime}}{\partial \ln \alpha}+\frac{\partial \ln \xi_{\perp}}{\partial \ln \alpha} \frac{\partial \ln N_{e}}{\partial \ln \xi_{\perp}} \bar{N}_{e}+\frac{\partial \ln \mathcal{A}}{\partial \ln \alpha}=0
$$

where $\bar{N}_{e}$ is the number of e-foldings of a sub-universe of maximum probability. If $\alpha^{\prime}$ is a power function of $\alpha$, the first term is of order unity. If $N_{e}$ and $\ln \mathcal{A} \sim-F$ are simple power functions of $\xi_{\perp}$ and $\alpha$, respectively, $\partial \ln N_{e} / \partial \ln \xi_{\perp}$ is also of order unity, and the last term is of order $-F(\alpha)$. Since we want our own sub-universe to be a highly probable one, $\bar{N}_{e}$ is approximately the number of e-foldings in our sub-universe, which is much larger than one. On the other hand, we would like our vacuum to be predicted inside the anthropic window, and hence $F(\alpha)$ should be of order unity. Thus, the second term must also be of order unity at the peak of the distribution, in spite of the large value of $N_{e}$. This is possible due to the sharp distribution, as long as

$$
\frac{\partial \ln \alpha}{\partial \ln \xi_{\perp}} \approx \bar{N}_{e}
$$

is satisfied. The more the sub-universe inflates, the sharper is the required behaviour of $\alpha(\xi)$.

\subsection{A Mass Threshold in Inflaton Decays}

After inflation the universe must reheat, so that the inflaton must decay. We assume that the inflaton mass scans $\left(\xi=m_{\phi}\right)$ and that $\mathcal{V}\left(m_{\phi}\right)$ (or the combination $I(\xi) \mathcal{V}(\xi)$ ) increases as $m_{\phi}$ decreases. As the inflaton mass $m_{\phi}$ is scanned, mass thresholds in the inflaton decay rate 


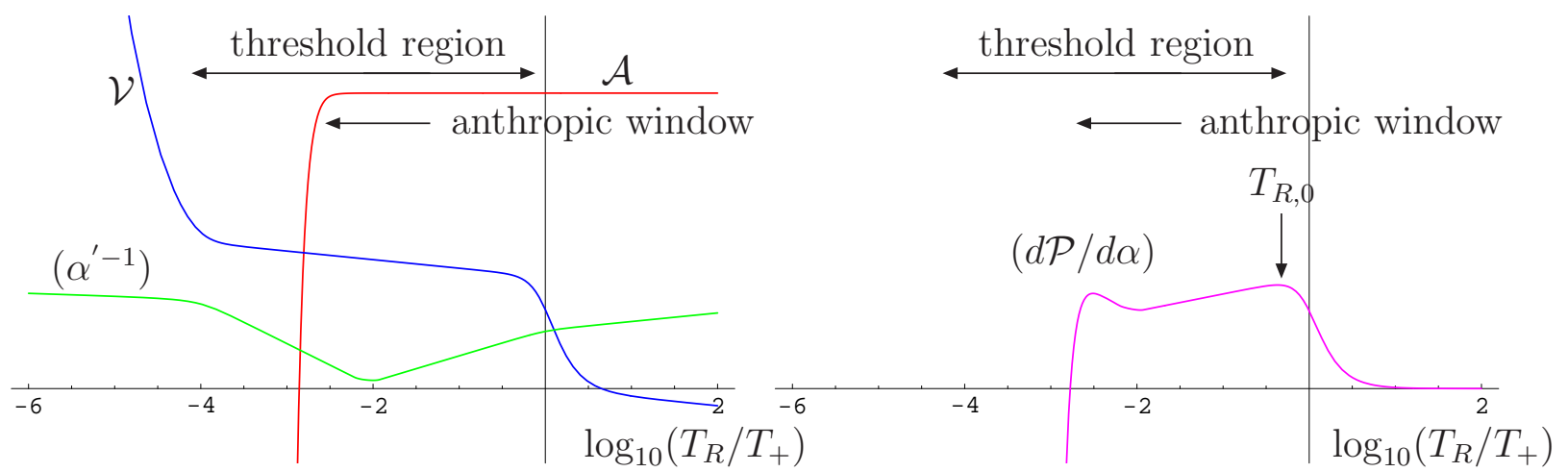

Figure 3: Left: various factors of the probability distribution, namely, $\mathcal{A}, \mathcal{V}$ and $(d \alpha / d \xi)^{-1}$, on a logarithmic scale. The normalization of each factor is arbitrary, and hence there is no importance to the absolute height. Right: combined probability distribution $d \mathcal{P} / d \alpha$. Note that the peak of the distribution is inside the anthropic window. [In this figure, the plateau of the volume factor in the threshold region corresponds to $\bar{N}_{e} \sim 10$. This is why the peak $T_{R, 0}$ is close to the upper end of the threshold region.]

automatically provide a sharp behaviour in the reheating temperature $T_{R}$ : thus we take $\alpha(\xi)$ to be a single relevant parameter, the reheating temperature $T_{R}\left(m_{\phi}\right)$. Suppose the inflaton $\phi$ decays dominantly to a pair of heavy fermions, $\phi \rightarrow \psi_{X}+\psi_{X}$, through the operator

$$
\mathcal{L}=\frac{1}{2} \lambda \phi \psi_{X} \psi_{X}+\text { h.c. }
$$

and that the universe is reheated by $\psi_{X}$ decaying to a fermion $\psi_{Y}$ and a scalar $Y$ through

$$
\mathcal{L}=\lambda^{\prime} \psi_{X} \psi_{Y} Y+\text { h.c.. }
$$

For $m_{\phi}>2 m_{X}$, the 2-body decay rate of the inflaton is

$$
\Gamma\left(\phi \rightarrow \psi_{X}+\psi_{X}\right)=\frac{1}{16 \pi} \lambda^{2} m_{\phi} \sqrt{\beta},
$$

where $\sqrt{\beta}$ describes the mass threshold, and $\beta \equiv 1-\left(2 m_{X} / m_{\phi}\right)^{2}$ decreases sharply as the inflaton mass $m_{\phi}$ approaches the threshold $2 m_{X}$ from above. The 2-body decay channel closes when $m_{\phi}<2 m_{X}$, forcing the inflaton to decay through a 3-body channel

$$
\phi \rightarrow \psi_{X}+\psi_{X}^{*} \rightarrow \psi_{X}+\psi_{Y}+Y,
$$

where $\psi_{X}^{*}$ is virtual, with a decay rate

$$
\Gamma\left(\phi \rightarrow \psi_{X}+\psi_{Y}+Y\right) \approx \frac{1}{16 \pi}\left(\frac{2 \lambda^{\prime 2}}{16 \pi^{2}}\right) \lambda^{2} \frac{m_{\phi}^{3}}{m_{X}^{2}}
$$


for $m_{X}<m_{\phi}<2 m_{X}$. The decay rate of the inflaton suddenly decreases by roughly $\left(\lambda^{\prime} / 4 \pi\right)^{2}$ as the inflaton crosses the threshold at $m_{\phi} \simeq 2 m_{X}$. Since the reheating temperature $T_{R}$ is given by $\sqrt{\Gamma M_{\mathrm{pl}}}$, it drops rapidly

$$
\begin{aligned}
\text { from } & T_{+} \equiv \min \left(\lambda, \lambda^{\prime}\right) \sqrt{m_{\phi} M_{\mathrm{pl}} / 16 \pi} \\
\text { to } T_{-} & \equiv \frac{\lambda \lambda^{\prime}}{4 \pi} \sqrt{m_{\phi} M_{\mathrm{pl}} / 16 \pi}
\end{aligned}
$$

as $m_{\phi}$ passes through the threshold at $2 m_{X}$, as shown in Fig. 2. This illustrates the origin of the sharp behaviour in $\alpha(\xi)$ : the late-time cosmology parameter $\alpha$ is $T_{R}$ and the high-energy parameter $\xi$ is the inflaton mass, $m_{\phi}$. Of course the idea in section 2.2 will work only when the anthropic factor depends exponentially onn $T_{R}$, so that we will be led to consider theories where the baryon asymmetry and/or the dark matter relic density depend on $T_{R}$.

Let us examine more explicitly how the averaged value of low-energy observables are determined in the example of $\alpha(\xi)=T_{R}\left(m_{\phi}\right)$ above. The threshold behaviour of $T_{R}$ is approximated ${ }^{8}$ by

$$
\left.T_{R}\left(m_{\phi}\right)\right|_{m_{\phi} \approx 2 m_{X}} \approx \begin{cases}T_{+} \beta^{1 / 4} & {\left[m_{\phi}>2 m_{X}\left(1+\lambda^{\prime 2} /(16 \pi)\right)\right],} \\ T_{-}|\beta|^{-1 / 4} & {\left[m_{\phi}<2 m_{X}\left(1-\lambda^{\prime 2} /(16 \pi)\right)\right] .}\end{cases}
$$

Here, we have assumed that the reheating process above the threshold is governed by the decay of the inflaton $\phi$, rather than the decay of $\psi_{X}$. We focus on the part of the threshold with $m_{\phi}>2 m_{X}$, and substitute $\alpha(\xi)=T_{R}\left(m_{\phi}\right)=\beta^{1 / 4} T_{+}$in (6) to give

$$
T_{R, 0} \approx \frac{T_{+}}{\bar{N}_{e}^{1 / 4}}
$$

for a highly probable universe, such as our own. This is an important result. Our mechanism requires the reheat temperature of our sub-universe to lie in the upper half ${ }^{9}$ of the threshold region, so that $T_{R, 0}>\sqrt{T_{+} T_{-}} \approx \sqrt{\max \left(\lambda, \lambda^{\prime}\right) /(4 \pi)} T_{+}$. However, even for a huge volume factor $\mathcal{V}$, this requirement is very mild: $\max \left(\lambda, \lambda^{\prime}\right)<4 \pi / \bar{N}_{e}^{1 / 2}$. In the following sections we find that a sufficient exponential suppression of the anthropic factor will provide a somewhat stronger constraint on $\max \left(\lambda, \lambda^{\prime}\right)$.

\footnotetext{
${ }^{8}$ The approximation of $T_{R}$ using 3-body decay also exhibits sharp behaviour because the virtual $\psi_{X}$ is almost on shell as $m_{\phi}$ approaches the threshold from below. The definition of $\beta$ is $2\left[\left(m_{\phi} / 2 m_{X}\right)-1\right]$ around the threshold.

${ }^{9}$ Here, we assume that the volume factor increases as the inflaton mass decreases, as in Fig. 3 There may be another peak in the lower half of the threshold region, as in Fig. 3. but it depends on all the power-law components of all the factors in the probability distribution whether this peak exists or not, and which peak is higher. We just ignore this peak in this article.
} 
It is instructive to see in more detail how the probability distribution is maximized in this example of a mass threshold in inflaton decays. Assuming there is no significant structure in $I\left(m_{\phi}\right) d m_{\phi}$ around $m_{\phi} \approx 2 m_{X}$, the initial distribution is approximated by

$$
I\left(m_{\phi}\right) d m_{\phi} \propto d\left(m_{\phi}-2 m_{X}\right) \propto\left(\frac{T_{R}}{T_{+}}\right)^{4} d \ln \left(T_{R} / T_{+}\right) .
$$

Meanwhile, assuming that the e-fold number $N_{e}\left(m_{\phi}\right)$ does not have significant structure around $m_{\phi} \simeq 2 m_{X}$, we adopt the Taylor expansion

$$
N_{e}\left(m_{\phi}\right) \approx N_{e}\left(2 m_{X}\right) \times\left(1-c\left(\frac{m_{\phi}-2 m_{X}}{2 m_{X}}\right)\right) \approx \bar{N}_{e}(1-c \beta),
$$

with an order one coefficient $c$. Here $N_{e}$ is assumed to increase as $m_{\phi}$ decreases (as in the case of landscapes of chaotic-inflation regions [6, 8, 9]), hence the minus sign. The volume expansion factor is now approximated by

$$
\mathcal{V}\left(m_{\phi}\right) \approx e^{3 \bar{N}_{e}} e^{-3 c \bar{N}_{e}\left(T_{R} / T_{+}\right)^{4}}
$$

The overall probability distribution (ignoring the contribution from the anthropic factor in the anthropic window) is

$$
\mathcal{V}\left(m_{\phi}\right) I\left(m_{\phi}\right) d m_{\phi} \approx e^{3 \bar{N}_{e}} e^{-3 c \bar{N}_{e}\left(T_{R} / T_{+}\right)^{4}}\left(\frac{T_{R}}{T_{+}}\right)^{4} d \ln \left(T_{R} / T_{+}\right)
$$

has a peak around $T_{R} / T_{+} \approx\left(1 / \bar{N}_{e}\right)^{1 / 4}$, reproducing (15). This is how the typical $T_{R}$ is determined from this distribution, and will be the reheating temperature of our sub-universe $T_{R, 0}$ if our vacuum is to be typical. The inflaton mass $m_{\phi}$ is predicted in this framework almost at the threshold $2 m_{X}$ with a deviation of order $\left(T_{R, 0} / T_{+}\right)^{4} \approx 1 / \bar{N}_{e}$, and the reheating temperature is suppressed by $\left(1 / \bar{N}_{e}\right)^{1 / 4}$ from the naive expectation $T_{+}$because the inflaton mass is very close to the threshold.

The above argument shows how the probability distribution can form a peak within the anthropic window. We need to further make sure that this is the only peak, or at least, the biggest peak in the probability distribution. For $m_{\phi} \ll m_{X}, T_{R}\left(m_{\phi}\right)$ is not a particularly sharp function. $T_{R}$ different by a factor of a few would be a result of $m_{\phi}$ different by a similar degree, which means the number of e-folding different by a similar degree. Increase of $N_{e}$ by a factor of 2 implies that the volume factor is multiplied by an extra factor of $e^{3 N_{e}}$, since $e^{3 N_{e} \times 2}=e^{3 N_{e}} e^{3 N_{e}}$. Thus, we still need something that counters this exponential growth of probability distribution, 
and we expect the anthropic factor to do this job; $\mathcal{A}(\alpha) \approx e^{-F(\alpha)}$ with $\alpha=T_{R}$ now. If both $N_{e}(\xi)$ and $F(\alpha(\xi))$ are approximated by simple power functions of $\xi=m_{\phi}$ below the threshold, or $T_{R}<T_{-}$, it is sufficient to make sure that

$$
F\left(T_{-}\right) \gtrsim \bar{N}_{e}
$$

and that $F\left(m_{\phi}\right)$ is increasing faster than $N_{e}\left(m_{\phi}\right)$ as $m_{\phi}$ decreases, in order to guarantee that there is no other peak outside this threshold region.

In the following sections, we discuss what kind of cosmological scenarios can provide such an exponential anthropic factor $\mathcal{A}\left(T_{R}\right) \approx e^{-F\left(T_{R}\right)}$.

Independently of particular realization of $\mathcal{A}\left(T_{R}\right)$, however, one can see the following. It follows from the requirement $F\left(T_{-}\right) \gtrsim \bar{N}_{e}$ that $T_{-} / T_{+}$is sufficiently small. This means that there is an upper limit on

$$
\frac{T_{-}}{T_{+}}=\frac{\max \left(\lambda, \lambda^{\prime}\right)}{4 \pi}
$$

and hence on

$$
\frac{T_{+}}{\sqrt{m_{\phi} M_{\mathrm{pl}} /(16 \pi)}}=\min \left(\lambda, \lambda^{\prime}\right) \leq \max \left(\lambda, \lambda^{\prime}\right) .
$$

Thus, the reheating temperature of our universe $T_{R, 0}$ given by (15) is also bounded from above, once $\bar{N}_{e}$ and $m_{\phi}$ are known. Although the upper bound itself depends on such issues as particular cosmological scenarios that determine $F\left(T_{R}\right), \bar{N}_{e}$, and $m_{\phi}$, yet it is generic that we have an upper bound on $T_{R, 0}$ for individual cosmological scenario. We see a particularly important consequence in the scenario in section 4

In this article, we consider the scanning of a single parameter of the inflaton potential that causes both $m_{\phi}$ and $N_{e}$ to vary. It is beyond the scope of this article to explore the possibility of solving the runaway problem for more complicated inflationary landscapes with more than one scanning inflationary parameters. We just note here that the scanning of multiple inflationary parameters generically leads to exponential behaviour in multiple directions on the space of observable parameters, requiring multiple anthropic reasons for exponential suppression in the number of observers.

Our analysis in this article is based on an assumption that only two parameters are scanned in a landscape: the cosmological constant and the mass of the inflaton. All other parameters are assumed not to be effectively scanned: some parameters may not be scanned in the landscape [23, 24], or some of the parameters are scanned in the landscape but have already been pinned down to the value of our sub-universe via some anthropic argument. The two parameter 
scanning of $\left(m_{\phi}, \Lambda^{4}\right)$ is certainly minimal in considering the runaway problem, and at least the first place to start.

\section{An Exponential Anthropic Factor}

The two crucial ingredients for solving the runaway problem are to have a sharp threshold behaviour of a relevant parameter as the inflaton mass changes, and to have the anthropic factor $\mathcal{A}$ decreasing exponentially with this relevant parameter - not just as a power law. Since we have seen in the previous section that the reheating temperature exhibits a sharp behaviour as the inflaton mass passes a threshold $2 m_{X}$, it would be sufficient if, in some range of parameters, $\mathcal{A}$ depends exponentially on $T_{R}$. In the following sections, we show how this occurs in some cosmological scenarios.

The basic idea is the following. The baryon symmetry $Y_{B} \equiv n_{B} / s$ decreases in some cosmological scenarios if $T_{R}$ decreases; thermal leptogenesis [25] is an example - there is insufficient production of right-handed neutrinos in the thermal plasma if $T_{R}$ is less than the mass of the lightest one. Although the number of baryons in a given comoving volume determines the maximum number of galaxies available, and hence contributes to the anthropic factor, the baryon asymmetry itself is usually only a power-law function of $T_{R}$, and is not enough to counter the exponential growth of the volume factor. Some other physics consideration is necessary in order for $T_{R}$ to be exponentially relevant to the anthropic factor.

After density perturbations grow during the era of matter domination, baryons begin to self-gravitate to form bound states. In order for these bound states to evolve into such highly non-linear structures as galaxies, the systems must release energy. When the number density of baryons is too low, the cooling through bremsstrahlung is so inefficient that the time scale for the cooling becomes longer than the lifetime of a proton. This observation leads to an exponentially suppressed anthropic factor, as shown below in more detail.

The baryon asymmetry remains constant in thermal leptogenesis as long as $T_{R}>M_{1}$, where $M_{1}$ is the mass of the lightest right-handed neutrino:

$$
Y_{B, 0} \equiv\left(\frac{n_{B}}{s}\right)_{T_{R}>M_{1}} \simeq 0.35 \times\left(\frac{\Gamma_{N_{1}}}{M_{1}^{2} / M_{\mathrm{pl}}}\right) \epsilon_{\mathrm{CP}}
$$

where $\Gamma_{N_{1}} /\left(M_{1}^{2} / M_{\mathrm{pl}}\right)$ is $n_{N_{1}} / s$, assuming out-of-equilibrium decay and ignoring $g_{*}$, the effective statistic degrees of freedom. The $\mathrm{CP}$ asymmetry $\epsilon_{\mathrm{CP}}$ is given by

$$
\epsilon_{\mathrm{CP}}=\frac{3}{16 \pi} \frac{\operatorname{Im}\left(\left(h_{1 \alpha} h_{j \alpha}^{*}\right)^{2} M_{1} / M_{j}\right)}{\left|h_{1 \alpha}\right|^{2}} .
$$


As $T_{R}$ drops below $M_{1}$, however, the baryon asymmetry from thermal leptogenesis is much less than (23), because leptogenesis stops before reheating completes. After the end of the slow roll era, the inflaton field oscillates and the temperature of the thermal plasma behaves as $T^{4} \sim T_{R}^{2} \rho_{\text {infl. }}^{1 / 2}$ [26. Thermal leptogenesis stops roughly when $T \sim M_{1}$, and the subsequent entropy production continues until $T \sim T_{R}$, diluting the lepton asymmetry by a factor of $\left(T_{R} / M_{1}\right)^{5}$ [26]. Furthermore, the number density of right-handed neutrinos is less than usual because

$$
\left(\frac{n_{N_{1}}}{s}\right)_{T=M_{1}} \sim \Gamma_{N_{1}} t_{T=M_{1}} \sim\left(\frac{\Gamma_{N_{1}}}{\rho_{\mathrm{infl}}^{1 / 2} / M_{\mathrm{pl}}}\right)_{T=M_{1}} \sim \frac{\Gamma_{N_{1}}}{\left(M_{1}^{2} / M_{\mathrm{pl}}\right)}\left(\frac{T_{R}}{M_{1}}\right)^{2} .
$$

Thus, the baryon asymmetry decreases as

$$
Y_{B} \simeq Y_{B, 0}\left(\frac{T_{R}}{M_{1}}\right)^{7}
$$

for $100 \mathrm{GeV}<T_{R}<M_{1}$. The baryon asymmetry goes down rapidly ${ }^{10}$ as $T_{R}$ decreases, and so does the number of Milky-Way type galaxies in a given comoving volume. However, since this is only a power law of $T_{R}$, this is not enough to produce the desired exponential anthropic factor.

Let us assume that the dark matter density of our universe does not depend on $T_{R}$ (when $T_{R}$ is in the range, say, above the electroweak scale). Thermal relics of stable particles that weigh of order $100 \mathrm{GeV}$ (WIMPs) are a good enough candidate. Under this assumption, the epoch of matter-radiation equality remains almost unchanged. On the other hand, a single Milky-Way type galaxy has a fixed ${ }^{11}$ baryonic mass, of order $M_{\text {gal. }} \sim\left(10^{11}-10^{12}\right) M_{\odot}$, and

\footnotetext{
${ }^{10} \mathrm{An}$ implicit assumption here is that the branching ratio of the inflaton decay to right-handed neutrinos is small enough. In particular, none of the particles $\psi_{X}, \psi_{Y}$ and $Y$ in the model of threshold behaviour in the previous section can be identified with the right-handed neutrinos.

${ }^{11}$ The total mass of a galaxy is an important parameter of environment for the existence of observers. At least, the gravitational potential of a galaxy must be deep enough so that a supernova explosion cannot blow up the galaxy. Furthermore, correlations have been found observationally between luminosity (and mass) and metalicity of galaxies, and between the metalicity and sizes and orbiting radii of planets [27]. Thus, there will be a band (or at least lower bound) in the mass of galaxies suitable for the existence of observers, once all other cosmological and particle-physics parameters are fixed. In our universe, it will contain $10^{11}-10^{12} M_{\odot}$, and the lower bound of the band will be no less than $10^{8}-10^{9} M_{\odot}$ - the constraint from the supernova explosion. The band (or the lower bound) itself will be different from ours in a sub-universe different from ours. For instance, the supernova

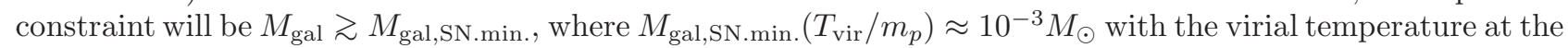
epoch runaway cooling starts. Constraints from the metalicity will require further astrophysical understanding. Because we do not know the anthropic band (lower bound) in universes quite different from ours, in this article we simply use the estimate $M_{\text {gal }} \sim\left(10^{11}-10^{12}\right) M_{\odot}$. These enormous astrophysical uncertainties, however, do not change one of our main claims that an exponential anthropic factor follows by requiring galaxies to form
} 
hence a galaxy has to collect baryons from much wider comoving volume in sub-universes having small baryon asymmetry. The galactic comoving scale enclosing a baryon of order $M_{\text {gal }}$ enters the horizon later if the baryon asymmetry is smaller. The horizon entry becomes as late as the epoch of matter-radiation equality if $Y_{B}$ were ${ }^{12}$ of order $\sim\left(M_{\text {gal }} T_{\text {eq }}^{3} / m_{p} M_{\text {pl }}^{3}\right) \sim$ $\left(10^{-15}-10^{-14}\right)\left(T_{e q} / 0.6 \mathrm{eV}\right)^{3}$. For $Y_{B}$ less than that, the galactic comoving scale would be proportional to $Y_{B}^{-1 / 3}$, the horizon-entry time to $Y_{B}^{-1}$, and the number density of the baryon asymmetry [photons] at horizon entry to $Y_{B}^{3}$ [to $\left.Y_{B}^{2}\right]$. This number density is diluted by $Q^{3}$ as the universe continues to expand, until the density perturbation of the galactic comoving scale goes non-linear. The density in over-dense regions increases further by of order 100 during the virialization process, and the evolution of gravitationally bound systems decouple from the expansion of the rest of the universe, ${ }^{13}$ giving proto-galaxies with

$$
\begin{aligned}
\rho & \approx 100 T_{e q}^{4}\left(Y_{B} / 10^{-14.5}\right)^{2}\left(0.6 \mathrm{eV} / T_{e q}\right)^{6} Q^{3} \\
n_{\gamma} & \approx\left(T_{e q} Q\right)^{3}\left(Y_{B} / 10^{-14.5}\right)^{2}\left(0.6 \mathrm{eV} / T_{e q}\right)^{6} \\
n_{B} & \approx 1000 n_{\gamma} Y_{B}
\end{aligned}
$$

at the epoch of virialization for $Y_{B}<\left(10^{-15}-10^{-14}\right)\left(T_{e q} / 0.6 \mathrm{eV}\right)^{3}$. Since the galactic comoving scale enters the horizon after matter-radiation equality for such low baryon asymmetry, the virial temperature of baryons is roughly given by [28]

$$
T_{\text {vir }} \sim m_{p} Q
$$

which is of order $10 \mathrm{keV}$ for $Q \sim 10^{-5}$.

For sub-universes with such a low baryon asymmetry, the condition for runaway cooling [29] of the proto-galaxies,

$$
\Gamma_{\text {cool }}>\left(G_{N} \rho\right)^{1 / 2}
$$

is not satisfied. Here, $\Gamma_{\text {cool }}$ is defined in terms of the energy-loss rate per particle

$$
d E_{\text {loss }} / d t=-\Gamma_{\text {cool }} T_{\text {vir }} .
$$

before protons decay, providing the anthropic band (lower bound) of galaxy masses does not decrease as fast as $Y_{B}^{3 / 2}$. Our quantitative predictions for the upper bound of the reheating temperature are not greatly affected either. Even if there are uncertainties of several orders of magnitude in the anthropic bound on $Y_{B}$ in (36) or [45), the limit on $T_{R}$ is changed very little because the baryon asymmetry depends so sensitively on $T_{R}$ in (26) or (44). See also section 5

${ }^{12}$ For $\Omega_{C D M} h^{2} \simeq 0.113$ and $\Omega_{B} h^{2} \simeq 0, T_{e q} \sim 0.63 \mathrm{eV}$.

${ }^{13}$ The cosmological constant has to be small enough, so that the universe remains matter dominated until this epoch. This only leads to a power-law suppression in the number of states in landscapes, and we ignore such a power-law contribution to the probablity distribution. 
Proto-galaxies can release energy by emitting photons. The contributions to $\Gamma_{\text {cool }}$ from the two relevant processes, namely bremsstrahlung $p+e \rightarrow p+e+\gamma$ and Compton scattering $e^{-}+\gamma \rightarrow e^{-}+\gamma$, are given by 30.

$$
\begin{aligned}
\Gamma_{\mathrm{Brems}} & \approx 9 \frac{\alpha^{3}}{m_{e}^{2}} \frac{n_{B}}{\sqrt{T_{\mathrm{vir}} / m_{e}}}, \\
\Gamma_{\mathrm{Comp}} & \approx \frac{2^{7} \pi^{3}}{810} \frac{\alpha^{2}}{m_{e}^{2}} \frac{T_{\gamma}^{4}}{m_{e}},
\end{aligned}
$$

where $T_{\gamma} \sim n_{\gamma}^{1 / 3}$ is the typical photon energy. To see that (31) is not satisfied, first note that Compton scattering is more efficient than bremsstrahlung right after virialization; second, the rate (34) does not satisfy (31).

Although [28] used the condition (31) as an anthropic criterion, the cooling condition (31) not being satisfied only implies ${ }^{14}$ that runaway cooling does not happen right after virialization. Instead, quasi-static cooling occurs on a time scale of order $\Gamma_{\text {cool }}^{-1}$, allowing gravitational contraction. During quasi-static cooling, the gravitationally bound system maintains virial equilibrium, and the Jeans mass remains equal to the total mass of the bound system [29]. Hierarchical cooling 31] does not occur. Thus, sub-structures of galaxies, such as clumps of molecular clouds or hydrogen-burning stars, are not formed during quasi-static cooling. As the quasi-static cooling proceeds, the system becomes more dense and hotter, ${ }^{15}$ and eventually the cooling condition (31) is satisfied, so that hierarchical cooling starts, and smaller structures begin to grow. ${ }^{16}$ Thus, we do not find it really safe to claim that the anthropic factor is exponentially suppressed whenever the cooling condition (31) is not satisfied.

The cooling time scale cannot be arbitrary long: ${ }^{17}$ galaxies must form before protons decay,

\footnotetext{
${ }^{14}$ The size of the cosmological constant determines the largest structures with order one density perturbations, but it is more appropriate to think that the upper bound on the cosmological constant is set by requiring that galaxies in the anthropic mass band be formed. Since the landscape picture prefers the largest anthropically allowed cosmological constant, it is possible that structures containing baryons of order $M_{\text {gal }}$ (or the lower anthropic mass bound for galaxies) are the largest. If so, the destruction of proto-galaxies through mutual gravitational interactions before cooling starts is no longer an anthropic problem [29. See, however, also footnote 17 and section 5

${ }^{15} \mathrm{At}$ an earlier stage of gravitational contraction, the source of gravitational potential energy is dominated by dark matter, and thus the temperature of baryons may not increase as in the standard $T_{B} \propto \rho_{B}^{1 / 3}$ relation. But after a baryonic core with $\rho_{B} \sim \rho_{\mathrm{CDM}}$ is formed, as a result of gravitational contraction of baryons, the temperature of the baryons (including electrons) begins to increase.

${ }^{16}$ It is the density at this epoch that should be used in evaluating the anthropic conditions in [32, ensuring that the halo not be too dense. See section 5

${ }^{17}$ Main-sequence stars and planetary systems around them are considered to have formed from cold clouds in galactic discs in our universe. Not all the particles are heated up to the virial temperature at the beginning
} 
which we assume happens with a lifetime of order $\tau_{p} \approx 10^{36}$ years:

$$
\Gamma_{\text {cool }}^{-1}<\tau_{p} \approx 10^{36} \mathrm{yrs}
$$

Since relativistic particles, namely photons, cannot be trapped in a gravitational potential well without sufficient scattering interactions, the number density of photons continues to decrease because of the expansion of the entire universe. On the other hand, the number density of protons remains constant in the virialized systems. Thus, the cooling time scale is set by the bremsstrahlung process. ${ }^{18}$ Using (28)-(30) and (33) in the anthropic condition (35), we see that

$$
\left(\frac{10^{-20}}{Y_{B}}\right)^{3}\left(\frac{2 \times 10^{-5}}{Q}\right)^{2.5} \lesssim 1
$$

If $Y_{B} \lesssim 10^{-18}$, however, the $\bar{p} p$ pair annihilation in the early universe is incomplete, ${ }^{19}$ so that $\bar{p}$ survive with an abundance of order $n_{\bar{p}} / s \sim 10^{-18}$, which, by charge conservation, is also the $e^{+}$abundance. The bremsstrahlung cooling rate should be evaluated by using $n_{p, \bar{p}}$ in (33), instead of $n_{B}$. But, since the cross section of $e^{+} e^{-}$pair annihilation is larger than that of $(e, p)$ bremsstrahlung, pair annihilation starts before cooling. As the number densities of electrons and positrons decrease, the $(e, p)$ bremsstrahlung cooling rate also decreases; the total energy carried by electrons (and positrons) is only $n_{e, \bar{e}} / n_{p, \bar{p}}$. In the end, only electrons are left with the abundance $n_{e}=n_{p}-n_{\bar{p}}$, and $\Gamma_{\text {Brems }}$ reduces to the one with $n_{B}$ again. Anti-protons annihilate with protons before proto-galaxies begin to cool. Thus, even for $Y_{B} \lesssim 10^{-18}$, the anthropic bound (36) is still valid. ${ }^{20}$

The anthropic bound (36) has to be satisfied by the actual density perturbation $Q$. Assuming that $Q$ follows a Gaussian distribution characterized by its standard deviation $\sigma$, we obtain the anthropic factor

$$
\mathcal{A}\left(\sigma, Y_{B}\right) \approx \int_{\left(Q / 2 \times 10^{-5}\right)>\left(10^{-20} / Y_{B}\right)^{6 / 5}} d Q e^{-(Q / \sigma)^{2}} \approx e^{-\left(10^{-20} / Y_{B}\right)^{12 / 5}\left(2 \times 10^{-5} / \sigma\right)^{2}} .
$$

of virialization, as argued in 33. But, if it takes too long time for proto-galaxies to cool, would-be discs may be heated and evaporate before runaway cooling starts. This anthropic condition is not taken into acount in section 3 and 4 See discussion in section $[5$

${ }^{18}$ Here, we assume that all the particles in proto-galaxies remain bounded gravitationally forever. However, this is not really correct; some particles evaporate from proto-galaxies before runaway cooling starts. See discussion in the appendix for the effects of evaporation.

${ }^{19} \mathrm{TW}$ thanks G. Perez for bringing this issue to our attention.

${ }^{20} \mathrm{We}$ assume that $e^{+}-e^{-}$and $p-\bar{p}$ pair annihilation emit photons and relativistic pions that freely escape from the gravitational potential of proto-galaxies. 
Since $Y_{B} \propto T_{R}^{7}$ for $T_{R}<M_{1}$ in the thermal leptogenesis scenario, this anthropic factor indeed depends exponentially on $T_{R}\left(m_{\phi}\right)$.

In order to see whether this exponentially small anthropic factor is small enough to contain the exponentially large volume factor, we need to be a little more specific about the inflationary landscape. We approximate the inflationary landscape by an ensemble of chaotic inflation potentials, with only one parameter $m_{\phi}$ to be scanned. The volume factor is given by $\mathcal{V} \approx e^{3 N_{e}}$ with $N_{e}\left(m_{\phi}\right) \approx M_{\mathrm{pl}} / m_{\phi}$, because the largest value of the inflaton field for classical slow-roll inflation is $\phi \approx M_{\mathrm{pl}}^{3 / 2} / m_{\phi}^{1 / 2}$. The amount of inflation increases as $m_{\phi}$ decreases, and thus the volume factor can, in principle, be countered by the threshold behaviour and the anthropic factor we have discussed. The asymptotic behaviour of the anthropic factor $\mathcal{A} \approx e^{-F\left(T_{R}\left(m_{\phi}\right)\right)}$ is given by

$$
F\left(T_{R}\left(m_{\phi}\right)\right) \propto Y_{B}^{-12 / 5} \sigma^{-2} \propto T_{R}^{-84 / 5} m_{\phi}^{-2} \propto m_{\phi}^{-27.2}
$$

where $\sigma \sim m_{\phi} / M_{\mathrm{pl}}$ for chaotic inflation and $T_{R} \propto \sqrt{\Gamma(\phi)} \propto m_{\phi}^{3 / 2}$ for $m_{X} \lesssim m_{\phi} \ll 2 m_{X}$ [see (11)]. Thus, as $m_{\phi}$ decreases, $F\left(T_{R}\left(m_{\phi}\right)\right)$ increases much faster than $N_{e}\left(m_{\phi}\right)$ and we conclude that the exponential anthropic factor from thermal leptogenesis is able to tame the volume factor from chaotic inflation.

The anthropic factor (37) should begin to decrease within the range of $T_{-}<T_{R}<T_{+}$covered by the threshold behaviour, so that the peak of the probability distribution is determined by the argument in the previous section. The requirement (20) on $T_{-}$now reads

$$
Y_{B}\left(T_{-}\right) \lesssim 10^{-20} \times\left(m_{\phi} / M_{\mathrm{pl}}\right)^{5 / 12} \approx 10^{-22}
$$

where the COBE normalization was used to determine the inflaton mass $m_{\phi} \simeq 10^{13} \mathrm{GeV}$ in chaotic inflation. This is translated into $T_{-} / M_{1} \lesssim 10^{-1.6}$, or equivalently, $\left(T_{-} / T_{+}\right) \lesssim$ $10^{-1.6} \bar{N}_{e}^{-1 / 4}\left(M_{1} / T_{R, 0}\right) \lesssim 10^{-3}\left(M_{1} / T_{R, 0}\right)$, with $\bar{N}_{e} \sim\left[2.4 \times 10^{18} \mathrm{GeV} / 10^{13} \mathrm{GeV}\right]$. Thus, the coupling constants for the reheating have to satisfy only a mild condition: $\max \left(\lambda, \lambda^{\prime}\right) \lesssim$ $10^{-2}\left(M_{1} / T_{R, 0}\right)$. Therefore, we obtain the upper bound on the reheating temperature as outlined in the previous section:

$$
T_{R, 0} \lesssim \sqrt{10^{12} \mathrm{GeVM}_{1}}
$$

and the lightest right-handed neutrino has to be somewhat lighter than $10^{12} \mathrm{GeV}$.

We have seen in this section that thermal leptogenesis can solve the runaway problem of chaotic inflation, with only a mild constraint on the coupling constants involved; $\lambda, \lambda^{\prime} \lesssim 10^{-2}$. We have also obtained some predictions; the upper bound on the lightest right-handed neutrino mass $M_{1}<10^{12} \mathrm{GeV}$, and the upper bound on the reheating temperature of our universe (40). 
Although these predictions are not directly testable in low-energy experiments, they provide extra constraints on the parameter space of thermal leptogenesis.

\section{Non-thermal Leptogenesis}

The key ingredient in section 2 is the existence of a heavy particle $\psi_{X}$, with mass $m_{X} \sim 10^{13}$ $\mathrm{GeV}$ that is not scanned in a landscape. This hypothetical particle $\psi_{X}$ realized the sharp threshold behaviour in the reheating temperature, and stopped the inflaton mass runaway at $2 m_{X}$. Can $\psi_{X}$ be related to other observable physics?

The tiny masses of left-handed neutrinos combined with the see-saw 34] mechanism suggest that right-handed neutrinos exist, with masses of order $10^{15} \mathrm{GeV}$ for order 1 Dirac Yukawa couplings and $10^{13} \mathrm{GeV}$ for order $10^{-1}$ Dirac Yukawa couplings. Thus, a natural question arises: can a right-handed neutrino be identified with the hypothetical particle $\psi_{X}$ ? If this idea works, one may be able to make further arguments in the future why $m_{X}$ is not scanned in landscapes by looking at how the masses of right-handed neutrinos arise. In this section, we show that one of the right-handed neutrinos can indeed be identified with the threshold particle. This means that the inflaton mass, and consequently the density perturbation, is determined by the physics of right-handed neutrinos.

Let us assume that the inflaton decays to a pair of right-handed neutrinos $N_{1}$ with a branching ratio very close to 1 via the interaction

$$
\mathcal{L}=\frac{1}{2} \lambda \phi N_{1} N_{1}+\text { h.c. }
$$

The right-handed neutrinos decay to a lepton $l$ and a Higgs scalar $h_{u}$ through

$$
\mathcal{L}=\lambda_{i \alpha}^{\prime} N_{i} l_{\alpha} h_{u}+\text { h.c. }
$$

These two operators are the same as (77) and (8), allowing exactly the same behaviour as in section 3. The inflaton mass is predicted to be $\simeq 2 m_{N_{1}}$, and the reheating temperature of our sub-universe is suppressed by roughly $1 / N_{e}^{1 / 4}$ relative to the reheating temperature naturally expected when the inflaton mass is safely above the threshold $2 m_{N_{1}}$.

An important difference from the scenario in the previous section, however, is that the baryon asymmetry does not decrease as fast as in (26). The lepton asymmetry from the thermal leptogenesis certainly goes down as in (26), but there is an extra contribution from the the leptogenesis in the decay of right-handed neutrinos produced by inflaton decay [25]. Its 
lepton asymmetry is given by

$$
Y_{B} \simeq 0.5 \frac{T_{R}}{m_{\phi}} \epsilon_{\mathrm{CP}} \equiv \tilde{Y}_{B, 0}\left(\frac{T_{R}}{T_{R, 0}}\right),
$$

and decreases very slowly as $T_{R}$ decreases. Thus, the lepton (or baryon) asymmetry is dominated by this contribution for low reheating temperatures. Since the baryon asymmetry still depends on $T_{R}$, the sharp behaviour of $T_{R}\left(m_{\phi}\right)$ can be used to solve the runaway problem. The lepton asymmetry, however, depends so weakly on $T_{R}$, as opposed to (26), that a baryon asymmetry as low as $Y_{B} \sim 10^{-22}$ is not achieved for $T_{R}$ larger than the electroweak scale. For reheating temperatures lower than the electroweak scale, the baryon asymmetry decreases much faster, i.e.,

$$
Y_{B} \approx \tilde{Y}_{B, 0}\left(\frac{100 \mathrm{GeV}}{T_{R, 0}}\right)\left(\frac{T_{R}}{100 \mathrm{GeV}}\right)^{5}
$$

because the sphaleron process stops after the temperature of the thermal plasma drops below $100 \mathrm{GeV}$, and the baryon asymmetry generated at high temperature is diluted by radiation produced from inflaton decay. Thus, the reheating temperature below the threshold, $T_{-}$, is likely to be lower than the electroweak scale, but not much less.

The anthropic bound (36) on $Y_{B}$ (and on the density perturbation $Q$ ) was based on an assumption that the dark matter energy density does not change, but, with a reheating temperature lower than the electroweak scale, this assumption may not hold. If cold dark matter is a thermal relic of a stable particle with mass of order $100 \mathrm{GeV}$ (WIMP dark matter), production after reheating may be highly suppressed. The relic number density is frozen when the plasma temperature is around $10 \mathrm{GeV}$, and is diluted by a factor of order $\left(T_{R} / 10 \mathrm{GeV}\right)^{5}$ due to the following entropy production from inflaton decay. Gravitinos produced from thermal scattering are also a candidate for dark matter in gauge-mediated supersymmetry breaking scenarios, and their number density also decreases as the reheating temperature decreases - not exactly in the same manner for $T_{R}$ above the electroweak scale, though.

For $T_{R}$ sufficiently less than $10 \mathrm{GeV}$ for WIMP dark matter, the dominant component of dark matter is left-handed neutrinos. The effect of a lower dark matter density is incorporated by taking $T_{e q}$ to be $m_{\nu} \approx 0.03 \mathrm{eV}$ in (27)-(29). Thus, the anthropic bound (36) is replaced by

$$
\left(\frac{10^{-21}}{Y_{B}}\right)^{3}\left(\frac{2 \times 10^{-5}}{Q}\right)^{2.5}<1 .
$$

Note that the galactic comoving scale enclosing a baryonic mass of $M_{\text {gal }}$ is longer than the free-streaming length of neutrinos for $Y_{B}$ as low as $10^{-21}$, and the neutrinos can be treated as 
cold dark matter in the earlier stage of galaxy formation. The same argument as in the previous section leads to an exponential cut off for low reheating temperature and small inflaton mass, and it is sufficient to take $T_{-}$so that

$$
Y_{B}\left(T_{-}\right) \lesssim 10^{-21} \times\left(m_{\phi} / M_{\mathrm{pl}}\right)^{5 / 12} \approx 10^{-24}
$$

Thus, $T_{-} \sim 1 \mathrm{GeV}$ is sufficient for $T_{R, 0} \sim 10^{5.5} \mathrm{GeV}$, and $T_{-} \sim 10 \mathrm{GeV}$ for $T_{R, 0} \sim 10^{10.5} \mathrm{GeV}$. Since $Y_{B}$ depends on $T_{R}$ quite sensitively in (44) for low $T_{R}$, the exponential anthropic factor in (37) is powerful enough to tame the volume factor of chaotic inflation. At the same time, one will notice that various uncertainties already mentioned in footnotes in the previous section do not lead to a large uncertainty in the constraint on $T_{-}$.

One can also see that the bound (45) (and (46)) is valid for gravitino dark matter as well, essentially because left-handed neutrinos become the dominant cold dark matter and the other components are small and no longer relevant.

Now we can derive the upper bound on the reheating temperature of our universe, using the constraint (46). Higher reheating temperature $T_{R, 0}$ (and higher $T_{+}$) requires larger coupling constant, because $T_{+} \approx \min \left(\lambda, \lambda_{i=1, \alpha}^{\prime}\right) \times 10^{15} \mathrm{GeV}$, where $m_{\phi} \sim 10^{13} \mathrm{GeV}$ is used for chaotic inflation. This means larger $T_{-} / T_{+} \sim \max \left(\lambda, \lambda^{\prime}\right) /(4 \pi)$. Since the constraint (46) sets an upper limit on $T_{-}, T_{R, 0}$ cannot be arbitrary high. Let us assume $\bar{N}_{e}^{1 / 4} \sim 10$. For $T_{R, 0} \sim 10^{7}$ $\mathrm{GeV}, T_{+} \sim 10^{8} \mathrm{GeV}$, which means that $\min \left(\lambda, \lambda_{i=1, \alpha}^{\prime}\right) \sim 10^{-7}$. The threshold behaviour of $T_{R}\left(m_{\phi}\right)$ can cover the range from $T_{+}$to $T_{-} \sim\left(\max \left(\lambda, \lambda^{\prime}\right) / 4 \pi\right) T_{+} \gtrsim 1 \mathrm{GeV}$. Thus, for $\lambda \sim \lambda_{i=1, \alpha}^{\prime} \sim 10^{-7}$, the anthropic factor is already sufficiently exponentially small at the lower end of the threshold. For $T_{R, 0} \sim 10^{8} \mathrm{GeV}, T_{-}$cannot be less than $100 \mathrm{GeV}$, and the anthropic factor is not exponentially small. The upper bound on the reheating temperature of our subuniverse, $T_{R, 0}$, is only slightly above $10^{7} \mathrm{GeV}$.

At the same time we obtain the upper bound on the Dirac Yukawa coupling constants of the lightest right-handed neutrinos: ${ }^{21}\left|\lambda_{i=1, \alpha}^{\prime}\right| \lesssim 10^{-7}$.

Leptogenesis through inflaton decay accounts for the baryon asymmetry of our universe $Y_{B, 0} \sim(0.4-0.9) \times 10^{-10}$ only when $T_{R, 0} \gtrsim 10^{6} \mathrm{GeV}$, since the CP asymmetry in (24) cannot be more than order one. Thus, we see that the range of reheating temperatures of our universe is narrowed down to one order of magnitude, $10^{6} \mathrm{GeV} \sim 10^{7} \mathrm{GeV}$. This means that the CP phase in leptogenesis is no less than of order 0.1 . If there is no significant accidental cancellation with other combinations of CP phases in the lepton sector, there is a chance that $\mathrm{CP}$ violation will

\footnotetext{
${ }^{21}$ Dirac Yukawa coupling constants of other right-handed neutrinos are not constrained. Thus, the CP asymmetry can be large enough to account for the lepton/baryon asymmetry of order $10^{-10}$.
} 
be seen in the next-generation of neutrino oscillation experiments.

The other prediction, $\left|\lambda_{i=1, \alpha}^{\prime}\right| \lesssim 10^{-7}$, implies that the mass matrix of left-handed neutrinos is virtually of rank 2 , if there are 3 right-handed neutrinos. Some predictions on the rate of neutrinoless double beta decay are available in the rank 2 case; see 35] for more information.

These predictions are based on chaotic inflation, where the inflaton mass is determined by the COBE normalization $m_{\phi} \simeq 10^{13} \mathrm{GeV}$. We immediately learn that one of the right-handed neutrinos has a mass $m_{\phi} / 2$.

With weak-scale supersymmetry breaking ${ }^{22}$ arising from gauge mediation, our prediction for the range of reheating temperatures $T_{R, 0}$ has a significant further implication. In these theories, the gravitino is the best dark matter candidate for a wide range of gravitino masses, $100 \mathrm{keV}-100 \mathrm{GeV}$. The relic density of gravitinos produced from the thermal plasma is given by 36

$$
\Omega_{3 / 2} h^{2} \simeq 0.2\left(\frac{T_{R}}{10^{6} \mathrm{GeV}}\right)\left(\frac{10 \mathrm{MeV}}{m_{3 / 2}}\right)\left(\frac{m_{\tilde{g}}(100 \mathrm{GeV})}{1 \mathrm{TeV}}\right)^{2},
$$

and depends on two totally unknown parameters $m_{3 / 2}$ and $T_{R}$. Only the ratio $\left(T_{R} / m_{3 / 2}\right)$ can be determined from the observed value of $\Omega h^{2} \simeq 0.113$. But, now that the reheating temperature is essentially predicted the gravitino mass is determined, $m_{3 / 2} \simeq 20 \mathrm{MeV}-200 \mathrm{MeV}$. This in turn determines the fundamental scale of supersymmetry breaking, $\sqrt{F} \approx 10^{8}-10^{9} \mathrm{GeV}$.

\section{Conclusions and Discussion}

If the fundamental theory allows more than one vacuum, and if many vacua are realized cosmologically, then cosmology may play an important role in choosing the low energy theory and its parameters. In inflationary landscapes, where models and parameters of inflation are scanned cosmologically, sub-universes with a small inflaton mass dominate the volume of the universe, making slow-roll inflation a probable consequence. The problem of inflationary landscapes [8, 9], however, is that the probability distribution tends to be exponentially sensitive to some late-time cosmological parameters, and hence does not correctly predict their observed values. We call this the inflation runaway problem.

Landscapes and many-universes are about the world outside the horizon of our universe, and we usually think that no sign of them can be seen. One also usually considers that the an-

\footnotetext{
${ }^{22}$ In supersymmetric theories, the Affleck-Dine mechanism is another way to generate the baryon asymmetry. However, a negative mass squared is generated only when the Kähler potential satisfies certain conditions. If a landscape supports a region where the conditions are satisfied, an extra consideration is necessary.
} 
thropic conditions depend on only a limited number of relevant parameters, most of which have already been measured, so that there are no predictions. Without a well-understood top-down tool to figure out the statistic distribution of vacua on a landscape, it seems almost impossible to determine theories or parameters that are yet to be tested in experiments. It is important to note, however, that inflationary landscapes have a generic prediction: the probability distribution is exponentially sensitive to some parameters. This is where the runaway problem comes from. The problem is so severe that there may be very few possible solutions, from which indications may be obtained for particle physics to be explored in the future.

Some ideas have already been proposed to evade this problem. For instance, eternal inflation of false-vacuum type or chaotic-type may strongly select only one inflationary region in a landscape [8, 9]. The observational consequences for the curvature of the universe and for lower multipoles of CMB spectrum are discussed in [19] and references therein. Maybe the fundamental theory of phsycis has an inflationary landscape carefully designed to avoid the volume factor exponentially sensitive to some parameters [8, 13]. If such landscapes involve chaotic type inflation, we may expect tensor perturbations. A fundamental cut off scale parametrically larger than the observed Planck scale may also have something to do with the apparent fine tuning problems of certain low-energy supersymmetric theories [38. An alternative is to have density perturbations generated by a curvaton or by modulated reheating [8, 9], whose observational consequences are discussed in the literature [11, 39, 13].

In this article, we have proposed a new solution to the runaway problem, which is connected to the particle physics of inflaton decay and baryogenesis. In spite of the exponential distribution from the inflationary landscapes, observables are predicted successfully in the middle of the allowed anthropic windows. The key ingredient is to distinguish between parameters describing inflation and those that are directly observed. The latter are not necessarily given by simple power-law functions of the former. For instance, the reheating temperature drops sharply as the inflaton mass approaches the threshold for two body decay. In this case, the inflationary landscape predicts the inflaton mass to be close to twice the mass of the threshold particle.

A sufficiently sharp threshold behaviour for $T_{R}$ requires small couplings in operators relevant to reheating, and hence a low $T_{R}$ in our universe that depends on the cosmological scenario: $T_{R} \lesssim 10^{12} \mathrm{GeV}$ in the thermal leptogenesis scenario of section [3] and $10^{6} \mathrm{GeV} \lesssim T_{R} \lesssim$ $10^{7} \mathrm{GeV}$ in the inflaton-decay leptogenesis scenario of section 4 . Chaotic inflation is assumed. In both sections, the anthropic limit was set by requiring that bremsstrahlung and runaway hierarchical cooling of proto-galaxies start before protons decay. Although we have ignored various uncertainties, the upper bound obtained on $T_{R}$ illustrates a completely new way to 
obtain information on particle cosmology from anthropic reasoning.

In section 4 the particle produced by inflaton decay was identified with a right-handed neutrino, allowing the inflaton mass, and some other parameters including the normalization of density perturbations, to be set by physics of the neutrino sector. This provides a mechanism for understanding the values of inflation parameters in terms of observable particle physics. In this particular scenario, there are several predictions that again demonstrate how this line of anthropic argument can lead to observable tests. One CP violating phase of the neutrino sector must be of order 0.1 or larger. The low energy neutrino mass matrix is forced to be essentially rank 2 , so that the rate for neutrinoless double beta decay will be constrained by a particular relationship involving the neutrino mixing angles and the observed neutrino masses. Furthermore, in supersymmetric theories with gauge mediation, dark matter is predicted to be gravitinos with mass in the range 20-200 MeV. This corresponds to a fundamental scale of supersymmetry breaking of $10^{8}-10^{9} \mathrm{GeV}$, which can be tested by precise measurements of the superpartner spectrum and by the nature of the decay of the next-to-lightest superpartner [37].

It should be noted that the above predictions are based on an assumption that the anthropic limit is set by the comparison between the rate of bremsstrahlung cooling and proton decay. When various other anthropic conditions are imposed, some of which are listed in footnotes in section 3 , the anthropic limit in the $Y_{B^{-}} Q$ plane may be stronger, and the upper bounds on $T_{R}$ may become weaker. Including other uncertainties in galaxy formation and in the anthropic conditions, the above predictions may be modified. There is no doubt that further studies in astrophysics will be important in making the predictions more precise and reliable. As a step in this direction, we discuss possible effects of particle evaporation from proto-galaxies in the appendix, concluding that the upper bound on $T_{R}$ will not be greatly changed, partly because, in scenarios in section 3 and $4 Y_{B}$ is highly sensitive to $T_{R}$.

As the astrophysics and anthropic conditions become better understood, the upper bounds of the reheating temperature will change numerically. But, the idea for stopping the runaway by a combination of threshold effects and exponential anthropic factors will survive; extra anthropic conditions would only strengthen our conclusion that the exponential distribution of inflationary landscapes can be tamed. No matter how sophisticated and complicated the astrophysical analysis becomes, the upper bound on the reheating temeperature persists as long as the threshold behaviour of the inflaton decay plays a crucial role. 


\section{Acknowledgements}

This work was supported in part by the Director, Office of Science, Office of High Energy and Nuclear Physics, of the US Department of Energy under Contract DE-AC03-76SF00098 and DE-FG03-91ER-40676, DE-AC02-05CH11231, in part by the National Science Foundation under grant PHY-00-98840 (L.H., T.W.), and in part by a Humboldt Research Award (T.T.Y.). We thank B. Feldstein and K.-I. Izawa for discussion. T.W. thanks the Miller Institute for Basic Research in Science. T.T.Y. thanks the DESY theory group for hospitality, where a part of this work was done.

\section{Appendix: Evaporation}

In proto-galaxies that cannot cool, some fraction of the baryons in the high-energy tail of the Boltzmann distribution have sufficient speed to escape, as noted in [40. We ignored this effect in the main text, and devote this appendix to investigating the extent to which the conclusions could be changed by evaporation.

There are two important points in discussing evaporation from proto-galaxies. One is that the evaporation of particles from isolated proto-galaxies results in their gravitational contraction [41]. The other is that the proto-galaxies are comprized of multiple components: dark matter particles and baryons (including electrons and possibly their anti-particles). We begin for simplicity with the evolution of proto-galaxies made purely of cold dark matter particles, and later extend the discussion to take account of baryons.

Nearly $1 \%$ of particles in the Maxwell-Boltzmann distribution have velocities larger than the escape velocity of proto-galaxies and hence evaporate. The time scale of evaporation is set by how quickly the lost population of particles in the high-energy tail of the Boltzmann distribution is restored by particle-particle interactions. Two-body interactions via gravity always exist, no matter what the nature of the dark matter particle, with an efficiency given by

$$
\Gamma_{\text {grav. }} \approx \frac{\left(G_{N} \rho\right)^{1 / 2}}{N_{D M}}
$$

where $N_{D M}$ is the typical number of dark matter particles in one proto-galaxy [41. Note that the number of baryons is very large, of order $N_{B} \sim 10^{68}$ for $M_{\text {gal }} \simeq 10^{11} M_{\odot}$, and $N_{D M}$ is extremely large if dark matter is composed of elementary particles with a moderate mass, such as $100 \mathrm{GeV}$, or even lighter. Thus, the gravitational encounter of two dark-matter particles is not effective within the time scale of bremsstrahlung cooling. 
When dark-matter particles have two-body non-gravitational scattering with a cross section $\sigma_{D D}$, the relaxation due to this scattering occurs at the rate $n_{D M} \sigma_{D D} v_{\mathrm{vir}}$, where $n_{D M}$ is the number density of dark-matter particles. The evaporation is roughly $1 \%$ of the relaxation rate, so that evaporation of dark-matter particle does not occur before bremsstrahlung cooling, if

$$
10^{-2} n_{\mathrm{DM}} \sigma_{D D} v_{\mathrm{vir}} \lesssim \Gamma_{\text {Brems. }}
$$

Using (27) and (33), this holds true as long as

$$
\sigma_{D D} \lesssim 10 \mathrm{pb}\left(\frac{m_{D M}}{100 \mathrm{GeV}}\right)\left(\frac{Y_{B}}{10^{-22}}\right)\left(\frac{2 \times 10^{-3}}{Q}\right)
$$

for dark matter with $\rho_{D M} / s \simeq 0.8 \mathrm{eV}$, and

$$
\sigma_{D D} \lesssim 10^{-22.5} \mathrm{~b}\left(\frac{Y_{B}}{10^{-24}}\right)\left(\frac{2 \times 10^{-3}}{Q}\right)
$$

for neutrino dark matter with $n_{\nu} / s \simeq 10^{-1.5}$. It is clear that cross sections for axion-axion scattering and neutrino-neutrino scattering satisfy (50) and (51), respectively, for the values of $Y_{B}$ given in the right-hand sides. Thus, the anthropic bounds (36) and (45) in section 3 and 14 are unaffected by the evaporation of dark matter in such cases.

While the inequality (50) is satisfied by most dark matter candidates, for example WIMPs, it could be violated when the interactions between dark matter particles are significant. In that case, the anthropic bound (36) in section 3 will be affected; dark-matter evaporation leads to the gravitational contraction of proto-galaxies, and then the baryon number density is also enhanced and bremsstrahlung cooling begins to work. At some point in the gravitational contraction due to evaporation and quasi-static bremsstrahlung cooling, runaway cooling starts. Thus, evaporation changes the estimate of the time scale before the hierarchical cooling starts, which is to be compared with the proton lifetime in (35). However, even for such dark matter candidates, the estimated anthropic bound in (36) is not greatly affected by the evaporation of dark matter particles, since unitarity implies that (500) is not violated by a large amount - not by of order $10^{10}$. When the anthropic bounds on $Y_{B}$ is translated to an upper bound on the reheating temperature $T_{R}$, the effect will be quite minor since $Y_{B}$ is very sensitive to $T_{R}$ in (26).

Let us now bring baryons into the discussion, ignoring the evaporation of dark matter particles. Baryons-baryon scattering has a large cross section and the Boltzmann distribution is repopulated much more quickly. We do not go into a detailed estimate of the evaporation rate of baryons, but an important point is that this evaporation is accompanied by a gravitational 
contraction of the baryons. As long as there is no efficient energy transfer between dark-matter particles and baryons, baryons fall into the potential well of proto-galaxies as the evaporation proceeds, so that the evaporation soon stops. Thus, the discussion in the main text is unaffected by the evaporation of baryons (and electrons) if the energy transfer between dark matter particles and baryons does not occur effeciently.

Consider the case that the dark-matter particle is lighter than the proton, for example the case of axions or neutrinos. Since the axion-nucleon and neutrino-nucleon scattering cross sections are so small, an inequality similar to (51) is safely satisfied. Thus, the energy transfer does not take place between axion or neutrino dark matter with baryons before bremsstrahlung cooling starts, and the conclusion of the previous paragraph holds.

When dark matter particle has a mass of order $100 \mathrm{GeV}-1 \mathrm{TeV}$, the kinetic energy of a proton typically increases by of order unity in a single collision with a dark-matter particle. Once a proton is kicked by that amount, it has a good chance to escape the gravitational potential. The typical rate for this to happen is $n_{D M} \sigma_{D N} v_{\text {vir }}$, which is to be compared with $\Gamma_{\text {Brems. }}$ Energy transfer from dark matter to baryons does not occur before bremsstrahlung cooling, if the dark-matter nucleon scattering cross section $\sigma_{D N}$ satisfies

$$
\sigma_{D N} \lesssim 0.1 \mathrm{pb}\left(\frac{m_{D M}}{100 \mathrm{GeV}}\right)\left(\frac{Y_{B}}{10^{-22}}\right)\left(\frac{2 \times 10^{-3}}{Q}\right) .
$$

Most candidates for dark matter have a scattering cross section with protons that is much smaller than $0.1 \mathrm{pb}$, and hence the discussion in section 3 is not affected by baryon evaporation from proto-galaxies.

In the rare cases that energy is transferred from dark matter to baryons, the baryons may evaporate efficiently from proto-galaxies since dark matter particles keep supplying energy to the baryons allowing them to escape. Since protons and electrons are light and minor components in proto-galaxies, the energy supply continues until proto-galaxies loose virtually all their protons and electrons. Thus, bremsstrahlung cooling should work in a time scale shorter than that of the energy transfer to baryons and evaporation of baryons. This anthropic condition sets a constraint in the $Y_{B}-Q$ plane in addition to the proton lifetime constraint (35). An argument can be constructed essentially in the same way as in the main text, and in the end, the main qualitative result is maintained even with evaporation: namely, an exponential anthropic factor results from the failure of structure formation, and an upper bound on the reheating temperature is required to make $Y_{B}\left(T_{-}\right)$small enough. Since (52) is not a stringent constraint, and since $Y_{B}$ is very sensitive to $T_{R}$, the upper bound on $T_{R}$ is not affected very much, even quantitatively. 
It should be noted, however, that the discussion so far, both in the appendix and hence in the main text, assumes that proto-galaxies are homogeneous, allowing a crude estimate of the impact of evaporation. The limit on the cross section, such as $10 \mathrm{pb}$, may change a little when one takes into account the concentration of particles at the centre of the proto-galaxies and the profiles of the matter distribution and gravitational potential. The limit may also change when additional anthropic conditions, such as those in footnotes in section 3, are taken into account, or when $M_{\text {gal }} \sim 10^{11} M_{\odot}$ is replaced by an anthropic lower bound on $M_{\text {gal }}$, yet to be determined precisely. Further study of such issues may change the argument in this appendix quantitatively, but the way of thinking will remain valid.

\section{References}

[1] e.g., J. Barrow and F. Tipler, "The Anthropic Cosmological Principle," Oxford University Press, 1986.

[2] E. Baum, "Zero Cosmological Constant From Minimum Action," Phys. Lett. B 133 (1983) 185 ;

S. W. Hawking, "The Cosmological Constant Is Probably Zero," Phys. Lett. B 134, 403 (1984);

S. R. Coleman, "Black Holes As Red Herrings: Topological Fluctuations And The Loss Of Quantum Coherence," Nucl. Phys. B 307, 867 (1988);

S. B. Giddings and A. Strominger, "Loss Of Incoherence And Determination Of Coupling Constants In Quantum Gravity," Nucl. Phys. B 307, 854 (1988).

[3] J. Garcia-Bellido, A. D. Linde and D. A. Linde, "Fluctuations of the gravitational constant in the inflationary Brans-Dicke cosmology," Phys. Rev. D 50, 730 (1994) arXiv:astro-ph/9312039;

A. Vilenkin, "Predictions from quantum cosmology," Phys. Rev. Lett. 74, 846 (1995) gr-qc/9406010;

J. Garcia-Bellido and A. D. Linde, "Stationarity of inflation and predictions of quantum cosmology," Phys. Rev. D 51, 429 (1995) arXiv:hep-th/9408023.

[4] S. Weinberg, "Anthropic Bound On The Cosmological Constant," Phys. Rev. Lett. 59, 2607 (1987);

G. Efstathiou, Mon. Not. R. astron. Soc. 274, L73 (1995); 
H. Martel, P. R. Shapiro and S. Weinberg, "Likely Values of the Cosmological Constant," Astrophys. J. 492, 29 (1998) arXiv:astro-ph/9701099.

[5] J. Garriga, M. Livio and A. Vilenkin, "The cosmological constant and the time of its dominance," Phys. Rev. D 61, 023503 (2000) arXiv:astro-ph/9906210;

S. A. Bludman, "Vacuum energy: If not now, then when?," Nucl. Phys. A 663, 865 (2000) arXiv:astro-ph/9907168.

[6] A. Vilenkin, in [3].

[7] A. D. Linde, "Chaotic Inflation," Phys. Lett. B 129, 177 (1983).

[8] B. Feldstein, L. J. Hall and T. Watari, "Density perturbations and the cosmological constant from inflationary landscapes," Phys. Rev. D 72, 123506 (2005) arXiv:hep-th/0506235.

[9] J. Garriga and A. Vilenkin, "Anthropic prediction for Lambda and the Q catastrophe," arXiv:hep-th/0508005.

[10] S. Webb, "If the Universe is Teeming with Aliens... Where Is Everybody?" Springer (2002).

[11] A. D. Linde and V. Mukhanov, "Nongaussian isocurvature perturbations from inflation," Phys. Rev. D 56, 535 (1997) arXiv:astro-ph/9610219;

K. Enqvist and M. S. Sloth, "Adiabatic CMB perturbations in pre big bang string cosmology," Nucl. Phys. B 626, 395 (2002) arXiv:hep-ph/0109214;

D. H. Lyth and D. Wands, "Generating the curvature perturbation without an inflaton," Phys. Lett. B 524, 5 (2002) arXiv:hep-ph/0110002;

T. Moroi and T. Takahashi, "Effects of cosmological moduli fields on cosmic microwave background," Phys. Lett. B 522, 215 (2001) [Erratum-ibid. B 539, 303 (2002)] arXiv:hep-ph/0110096.

[12] G. Dvali, A. Gruzinov and M. Zaldarriaga, "A new mechanism for generating density perturbations from inflation," Phys. Rev. D 69, 023505 (2004) arXiv:astro-ph/0303591; L. Kofman, "Probing string theory with modulated cosmological fluctuations," arXiv: astro-ph/0303614

[13] A. Linde and V. Mukhanov, "The curvaton web," arXiv:astro-ph/0511736.

[14] R. Bousso and J. Polchinski, "Quantization of four-form fluxes and dynamical neutralization of the cosmological constant," JHEP 0006, 006 (2000) arXiv:hep-th/0004134;

M. R. Douglas, "The statistics of string / M theory vacua," JHEP 0305, 046 (2003) arXiv:hep-th/0303194; 
S. Ashok and M. R. Douglas, "Counting flux vacua," JHEP 0401, 060 (2004) arXiv:hep-th/0307049;

T. Banks, M. Dine and E. Gorbatov, "Is there a string theory landscape?," JHEP 0408, 058 (2004) arXiv:hep-th/0309170;

F. Denef and M. R. Douglas, "Distributions of flux vacua," JHEP 0405, 072 (2004) arXiv:hep-th/0404116;

A. Giryavets, S. Kachru and P. K. Tripathy, "On the taxonomy of flux vacua," JHEP 0408, 002 (2004) arXiv:hep-th/0404243;

O. DeWolfe, A. Giryavets, S. Kachru and W. Taylor, "Enumerating flux vacua with enhanced symmetries," JHEP 0502, 037 (2005) arXiv:hep-th/0411061.

[15] A. D. Linde, "Eternal Chaotic Inflation," Mod. Phys. Lett. A 1, 81 (1986), "Eternally Existing Selfreproducing Chaotic Inflationary Universe," Phys. Lett. B 175, 395 (1986).

[16] A. D. Linde and A. Mezhlumian, "Stationary universe," Phys. Lett. B 307, 25 (1993) arXiv:gr-qc/9304015;

A. D. Linde, D. A. Linde and A. Mezhlumian, "From the Big Bang theory to the theory of a stationary universe," Phys. Rev. D 49, 1783 (1994) arXiv:gr-qc/9306035.

[17] P. J. Steinhardt, in The Very Early Universe, Proceedings of the Nuffield Workshop, Cambridge, 1982, eds: G. W. Gibbons, S. W. Hawking, and S. T. C. Siklos (Cambridge University Press,1983), pp.251;

A. Vilenkin, "The Birth Of Inflationary Universes," Phys. Rev. D 27, 2848 (1983).

[18] L. Susskind, "The anthropic landscape of string theory," arXiv:hep-th/0302219.

[19] B. Freivogel, M. Kleban, M. R. Martinez and L. Susskind, "Observational consequences of a landscape," arXiv:hep-th/0505232.

[20] A. Vilenkin, "Unambiguous probabilities in an eternally inflating universe," Phys. Rev. Lett. 81, 5501 (1998) arXiv:hep-th/9806185;

V. Vanchurin, A. Vilenkin and S. Winitzki, "Predictability crisis in inflationary cosmology and its resolution," Phys. Rev. D 61, 083507 (2000) arXiv:gr-qc/9905097;

J. Garriga and A. Vilenkin, "A prescription for probabilities in eternal inflation," Phys. Rev. D 64, 023507 (2001) arXiv:gr-qc/0102090;

M. Tegmark, "What does inflation really predict?," arXiv:astro-ph/0410281

A. H. Guth, "Inflation and eternal inflation," Phys. Rept. 333, 555 (2000) arXiv:astro-ph/0002156. 
[21] J. Garriga, D. Schwartz-Perlov, A. Vilenkin and S. Winitzki, "Probabilities in the inflationary multiverse," arXiv:hep-th/0509184;

R. Easther, E. A. Lim and M. R. Martin, "Counting pockets with world lines in eternal inflation," arXiv:astro-ph/0511233.

[22] H. Martel, P. R. Shapiro and S. Weinberg in [4].

[23] N. Arkani-Hamed, S. Dimopoulos and S. Kachru, "Predictive landscapes and new physics at a TeV," arXiv:hep-th/0501082.

[24] B. S. Acharya, F. Denef and R. Valandro, "Statistics of M theory vacua," arXiv:hep-th/0502060.

[25] for a review on leptogenesis, see W. Buchmuller, R. D. Peccei and T. Yanagida, "Leptogenesis as the origin of matter," arXiv:hep-ph/0502169.

[26] E. Kolb, M. Turner, "The Early Universe," Addison-Wesley, 1990.

[27] N. C. Santos, G. Israelian, M. Mayor, R. Rebolo, and S. Udry, "Statistical properties of exoplanets. II. Metalicity, orbital parameters, and space velocities," Astron. \& Astrophys. 398363 (2003);

C. Laws, G. Gonzalez, K. M. Walker, S. Tyagi, J. Dodsworth, K. Snider, and N. B. Suntzeff, "Parent Stars of Extrasolar Planets. VII. New Abundance Analyses of 30 Systems," Astrophys. J. 1252664 (2003);

G. Gonzalez, D. Brownlee, and P. Ward, "The Galactic Habitable Zone: Galactic Chemical Evolution," Icarus, 152, 185 (2001).

[28] M. Tegmark and M. J. Rees, "Why is the CMB fluctuation level $10^{-5}$ ?," Astrophys. J. 499, 526 (1998) arXiv:astro-ph/9709058.

[29] M. Rees, J. Ostriker, "Cooling, dynamics and fragmentation of massive gas clouds: clues to the masses and radii of galaxies and clusters," Mon. Not. R. astr. Soc. 179541 (1977).

[30] K. Lang, “Astrophysical formulae," Springer-Verlag (1974);

M. Longair, "High Energy Astrophysics" Cambridge University Press (1981).

[31] F. Hoyle, "On the fragmentation of gas clouds into galaxies and stars," Astrophys. J. 113 513 (1953);

M. Rees, "Opacity-limited hierarchical fragmentation and the masses of proto stars," Mon. Not. R. astr. Soc. 176483 (1976).

[32] M. Tegmark and M. Rees in [28];

E. M. Leitch, and G. Vasisht, "Mass extinctions and the sun's encounter with spiral arms." 
New Astronomy, 351 (1998) astro-ph/9802174;

J. Garriga and A. Vilenkin, "Testable anthropic predictions for dark energy," Phys. Rev. D 67, 043503 (2003) arXiv:astro-ph/0210358;

M. Tegmark, A. Aguirre, M. Rees and F. Wilczek, "Dimensionless constants, cosmology and other dark matters," arXiv:astro-ph/0511774.

[33] Ya. B. Zeldovich, "Gravitational Instability: An Approximate Theory for Large Density Perturbations," Astron. \& Astrophys. 584 (1970);

R. A. Sunyaev and Ya. B. Zeldovich, "Formation of Clusters of Galaxies; Protocluster Fragmentation and Intergalactic Gas Heating," Astron. \& Astrophys. 20189 (1972).

[34] T. Yanagida, in Proc. Workshop on the Unified Theory and the Baryon Number in the Universe, Tsukuba, 1979, eds. O. Sawada and S. Sugamoto, Report KEK-79-18 (1979);

M. Gell-mann, P. Ramond and R. Slansky, in "Supergravity" North-Holland, Amsterdam, 1979, eds. D.Z. Freedman and P. van Nieuwenhuizen.

[35] M. Fujii, K. Hamaguchi and T. Yanagida, "Predictions on the neutrinoless double beta decay from the leptogenesis via the LH(u) flat direction," Phys. Lett. B 538, 107 (2002) arXiv:hep-ph/0203189;

G. C. Branco, R. Gonzalez Felipe, F. R. Joaquim and T. Yanagida, "Removing ambiguities in the neutrino mass matrix," Phys. Lett. B 562, 265 (2003) arXiv:hep-ph/0212341.

[36] M. Bolz, A. Brandenburg and W. Buchmuller, "Thermal production of gravitinos," Nucl. Phys. B 606, 518 (2001) arXiv:hep-ph/0012052.

[37] W. Buchmuller, K. Hamaguchi, M. Ratz and T. Yanagida, "Supergravity at colliders," Phys. Lett. B 588, 90 (2004) arXiv:hep-ph/0402179, "Gravitino and goldstino at colliders," arXiv:hep-ph/0403203.

[38] M. Ibe, K. I. Izawa and T. Yanagida, "Realization of minimal supergravity," Phys. Rev. D 71, 035005 (2005) arXiv:hep-ph/0409203;

M. Ibe, T. Moroi and T. Yanagida, "Dark matter and baryon asymmetry of the universe in large-cutoff supergravity," Phys. Lett. B 620, 9 (2005) arXiv:hep-ph/0502074.

[39] M. Zaldarriaga, "Non-Gaussianities in models with a varying inflaton decay rate," Phys. Rev. D 69, 043508 (2004) arXiv:astro-ph/0306006;

D. Babich, P. Creminelli and M. Zaldarriaga, "The shape of non-Gaussianities," JCAP 0408, 009 (2004) arXiv:astro-ph/0405356.

[40] M. Tegmark, A. Aguirre, M. Rees and F. Wilczek in [32]. 
[41] V. A. Ambarzumian, Ann. Leningrad State U., No. 22 (1938);

L. Spitzer Jr., "The stability of isolated clusters," Mon. Not. R. astron. Soc. 100396 (1940);

S. Chandrasekhar, "Principles of stellar dynamics," University of Chicago Press (1942);

L. Spitzer, Jr., "Distribution of galactic clusters," Ap. J. 12717 (1958);

L. Spitzer, Jr. and R. Härm, "Evaporation of stars from isolated clusters," Ap. J. 127544 (1958);

I. King, "The escape of stars from clusters III," Astron. J. 63465 (1958). 